\title{
Taxonomy and phylogenetic appraisal of Montagnula jonesii sp. nov. (Didymosphaeriaceae, Pleosporales)
}

\section{Tennakoon $\mathrm{DS}^{1,2,3,4}$, Hyde $\mathrm{KD}^{1,2,3,4}$, Wanasinghe $\mathrm{DN}^{1,2,3,4}$, Bahkali $\mathrm{AH}^{5}$, Camporesi $E^{6,7}$, Khan $S^{1,8}$ and Phookamsak $R^{1,2,3}$}

\footnotetext{
${ }^{1}$ World Agro Forestry Centre, East and Central Asia, 132 Lanhei Road, Kunming 650201, Yunnan China

${ }^{2}$ Key Laboratory for Plant Biodiversity and Biogeography of East Asia (KLPB), Kunming Institute of Botany, Chinese Academy of Science, Kunming 650201, Yunnan China

${ }^{3}$ Center of Excellence in Fungal Research, Mae Fah Luang University, Chiang Rai, 57100, Thailand

${ }^{4}$ School of Science, Mae Fah Luang University, Chiang Rai, 57100, Thailand

${ }^{5}$ Department of Botany and Microbiology, King Saud University, Riyadh, Saudi Arabia

${ }^{6}$ A.M.B. Gruppo Micologico Forlivese "Antonio Cicognani”, Via Roma 18, Forli, Italy; A.M.B. Circolo Micologico "Giovanni Carini”, C.P. 314, Brescia, Italy

${ }^{7}$ Società per gli Studi Naturalistici della Romagna, C.P. 144, Bagnacavallo (RA), Italy

${ }^{8}$ Key Laboratory of Economic Plants and Biotechnology, Kunming Institute of Botany, Chinese Academy of Sciences; Kunming 650201, Yunnan China.
}

Tennakoon DS, Hyde KD, Wanasinghe DN, Bahkali AH, Camporesi E, Khan S, Phookamsak R 2016 - Taxonomy and phylogenetic appraisal of Montagnula jonesii sp. nov. (Didymosphaeriaceae, Pleosporales). Mycosphere 7 (9), 1346-1356, Doi 10.5943/mycosphere/7/9/8

\begin{abstract}
A saprobic member of Dothideomycetes was collected from dead branches of Fagus sylvatica in Italy. Morphology coupled with combined gene analysis of LSU, SSU, ITS and tef1- $\alpha$ sequence data, showed it to be a novel Montagnula species, which is introduced in this paper. Montagnula jonesii sp. nov. differs from other Montagnula species in having immersed, brown ascomata and ellipsoidal to fusiform, 3-septate ascospores with rounded ends and prominent guttules in each cell and is enlarged at the second cell from the apex. The new species is compared with other Montagnula species and a comprehensive description and micrographs are provided.
\end{abstract}

Key words - Dothideomycetes - Morphology - New species

\section{Introduction}

Didymosphaeriaceae is an important family in Pleosporales, Dothideomycetes (Aptroot 1995, Hyde et al. 2013, Ariyawansa et al. 2014a, b, Liu et al. 2015, Wanasinghe et al. 2016). Munk (1953) introduced Didymosphaeriaceae and typified the family by Didymosphaeria Fuckel with D. epidermidis as the type species. Didymosphaeriaceae is characterized by brown, thick-walled, 1septate ascospores and trabeculate pseudoparaphyses, which anastomose above the asci in a gelatinous matrix (Aptroot 1995, Hyde et al. 2013, Ariyawansa et al. 2014a, b). The members of Didymosphaeriaceae play a vital role as saprobes, endophytes and pathogens of plant substrates (Aptroot 1995, Ariyawansa et al. 2014a, Liu et al. 2015, Wanasinghe et al. 2016). Ariyawansa et al. (2014a) discussed the confusion surrounding genera of Didymosphaeriaceae and mentioned that the family appears to be a distinct family of Pleosporales based on the morphological considering, but the molecular data could not be resolved its phylogenetic placement as the distinct family from 
Montagnulaceae. The representative species Didymosphaeria rubi-ulmifolii which was introduced by Ariyawansa et al. (2014a), clustered within the Montagnulaceae as a separate genus. Hence, Ariyawansa et al. (2014a) showed that Montagnulaceae and Didymosphaeriaceae are synonyms and thus, Ariyawansa et al. (2014b) synonymized Montagnulaceae under Didymosphaeriaceae based on priority of the oldest name. Ariyawansa et al. (2014b) re-circumscribed genera in Didymosphaeriaceae and accepted 16 genera in this family. Wijayawardene et al. (2014a, b) introduced another two asexual genera in family viz. Paracamarosporium and Pseudocamarosporium. Furthermore, Crous et al. (2015a, b) introduced Verrucoconiothyrium and Xenocamarosporium and Ariyawansa et al. (2015) referred Austropleospora and Pseudopithomyces to Didymosphaeriaceae. Wanasinghe et al. (2016) introduced Laburnicola and Paramassariosphaeria to the family and thus 24 genera are presently accepted in Didymosphaeriaceae.

The genus Montagnula was introduced by Berlese (1896) to accommodate M. infernalis (Niessl) Berl. and M. gigantean (Mont.) Berl. based on the morphology and phylogeny, Ariyawansa (2014b) placed Montagnula in Didymosphaeriaceae. The genus is characterized by globose or sphaerical, immersed ascomata with a clypeus, claviform asci, fusoid or ellipsoid ascospores with transverse septa and one or more longitudinal septa (Barr 1990, Ariyawansa et al. 2014b). There have been several recent studies on the taxonomy of Montagnula with introducing novel species (Table 1). Presently, there are 32 epithets for Montagnula (Index Fungorum 2016).

The aim of this study is to introduce a new species, Montagnula jonesii. Maximumlikelihood (ML), maximum-parsimony (MP) and Bayesian analyses (BI) of combined LSU, SSU, ITS and tef1- $\alpha$ sequence data clearly showed this species grouped in Montagnula (99\% ML, 70\% MP and 0.99 PP support, Fig. 1). The new species is described, illustrated and compared with similar taxa.

Table 1. Recorded Montagnula species in recent studies (2015-2016)

\begin{tabular}{lll}
\hline Montagnula species & Authority & Reference \\
\hline M. bellevaliae & Wanasinghe, Camporesi, E.B.G. Jones \&K.D. Hyde & Hongsanan et al. (2015) \\
M. scabiosae & Wanasinghe, Camporesi, E.B.G. Jones \& K.D. Hyde & Hongsanan et al. (2015) \\
M. graminicola & Chethana, Thambugala, Camporesi \& K.D. Hyde & Liu et al. (2015) \\
M. saikhuensis & Wanasinghe, E.B.G. Jones \& K.D. Hyde & Wanasinghe et al. (2016) \\
M. cirsii & Qing Tian, Camporesi \& K.D. Hyde & Hyde et al. (2016) \\
\hline
\end{tabular}

\section{Materials \& methods}

Sample collection, morphological studies and isolation

Fresh specimens were collected from Arezzo (AR) Province in Italy. Specimens were taken to the laboratory in zip lock bags and observed with a JNOEC JSZ4 stereomicroscope. Ascomata and ascospores were examined with an OLYMPUS SZ61 compound microscope. Sections of the fruiting structures were mounted in water for microscopic studies and photomicrography. Images were taken using a Nikon ECLIPSE 80i compound microscope with a Canon EOS 600D digital camera. Permanent slides were prepared by mounting fungal material in lactoglycerol and sealed by applying nail-polish around the margins of cover slips. All measurements were calculated using Tarosoft Image Frame work program (IFW) and images used for figures processed with Adobe Photoshop CS3 Extended version 10.0 software (Adobe Systems, USA).

The specimens were deposited in the Mae Fah Luang University Herbarium (MFLU), Chiang Rai, Thailand and the herbarium of Cryptogams Kunming Institute of Botany Academia Sinica (KUN-HKAS), Yunnan, China. Living cultures were deposited in Mae Fah Luang University Culture Collection (MFLUCC) and Kunming Institute of Botany Culture Collection 
(KUMCC). Faces of Fungi and Index Fungorum numbers are registered as described in Jayasiri et al. (2015) and Index Fungorum (2016).

\section{DNA extraction and PCR amplification}

Genomic DNA was extracted from mycelium using Biospin fungus Genomic DNA extraction kit (BioFlux ${ }^{\circledR}$, Hangzhou, P. R. China) following the manufacturer's protocol. The DNA product was kept at $4{ }^{\circ} \mathrm{C}$ for the DNA amplification and maintained at $-20{ }^{\circ} \mathrm{C}$ for long term storage. The DNA amplification was carried out by polymerase chain reaction (PCR) using four genes, the large subunit (28S, LSU), small subunit (18S, SSU), internal transcribed spacers (ITS1, 5.8S, ITS2) and translation elongation factor 1-alpha gene (tef1- $\alpha$ ). The LSU gene was amplified by using the primers LROR and LR5 (Vilgalys \& Hester 1990, Liu et al. 1999, Sung et al. 2007), SSU gene was amplified using the primers NS1 and NS4 (White et al. 1990), nuclear ITS was amplified by using the primers ITS5 and ITS4 (White et al. 1990) and tef1- $\alpha$ gene was amplified using the primers EF1-983F and EF1-2218R (Rehner et al. 2001). The amplification reactions were performed in $25 \mu 1$ of total reaction which contained $9.5 \mu 1$ of sterilized water, $12.5 \mu 1$ of $2 \times$ Power Taq PCR MasterMix (Bioteke Co., China), $1 \mu$ l of each forward and reverse primers and $1 \mu$ of DNA template. The polymerase chain reaction (PCR) thermal cycle program for LSU, SSU, ITS and tef1- $\alpha$ genes amplification were provided as: initially $95^{\circ} \mathrm{C}$ for $3 \mathrm{mins}$, followed by 35 cycles of denaturation at $95{ }^{\circ} \mathrm{C}$ for $30 \mathrm{sec}$, annealing at $55^{\circ} \mathrm{C}$ for $50 \mathrm{sec}$, elongation at $72{ }^{\circ} \mathrm{C}$ for $30 \mathrm{sec}$ and final extension at $72{ }^{\circ} \mathrm{C}$ for 10 mins. The quality of PCR products were checked on $1 \%$ agarose gel electrophoresis stained with ethidium bromide. PCR products were purified and sequenced by Sangon Biotech (Shanghai) Co., Ltd, China. Nucleotide sequences were deposited in GenBank (Table 2).

\section{Sequencing and alignment}

Phylogenetic analysis used combined LSU, SSU, ITS and tef1- $\alpha$ sequence data and other related sequences used in the analyses (Table 2) were obtained from GenBank (http://www.ncbi.nlm.nih.gov/) based on recently published data (Hyde et al. 2016, Wanasinghe et al. 2016) and BLAST searches (https://blast.ncbi.nlm.nih.gov/Blast.cgi). The combined dataset consists of 72 sequences including our newly generated sequences. Pleospora herbarum (CBS 191.86, IT 956) and Pleospora tarda (CBS 714.68) were selected as the outgroup taxa. The multiple alignments were automatically aligned by MAFFT v. 7 at the web server (http://mafft.cbrc.jp/alignment/server; 2016). Alignments were refined where necessary and combined sequence alignments were obtained by using BioEdit v. 7.0.5.2 (Hall 1999).

\section{Phylogenetic analysis}

Maximum parsimony analysis (MP) was performed using PAUP (Phylogenetic Analysis Using Parsimony) version 4.0b10 (Swofford 2002), with parameters as described in Wanasinghe et al. (2014). Descriptive tree statistics for parsimony (Tree Length [TL], Consistency Index [CI], Retention Index [RI], Relative Consistency Index [RC] and Homoplasy Index [HI]) were calculated for trees generated under different optimality criteria. The Kishino-Hasegawa tests (Kishino \& Hasegawa 1989) were performed to determine whether the trees inferred under different optimality criteria were significantly different.

Maximum likelihood analysis was performed by RAxML v.7.2.8 (Stamatakis 2010) implemented in RaxmlGUI 1.3 (Silvestro \& Michalak 2012). Bootstrap support for the branches was generated with 1000 replicates.

The model of evolution was estimated by using MrModeltest 2.2 (Nylander 2004). A Bayesian analysis was conducted with MrBayes v. 3.1.2 (Huelsenbeck \& Ronqvist 2001) to evaluate Posterior probabilities (PP) (Rannala \& Yang 1996, Zhaxybayeva \& Gogarten 2002) by Markov Chain Monte Carlo sampling (BMCMC). Six simultaneous Markov chains were run for $3,000,000$ generations and trees were sampled every $100^{\text {th }}$ generations. The first 3000 trees representing the burn-in phase of the analyses were discarded and the remaining 27,000 (Post 
burning) trees used for calculating posterior probabilities (PP) in the majority rule consensus tree (Cai et al. 2006, Liu et al. 2012). Phylograms were visualized with FigTree v1.4.0 program (Rambaut 2012) and annotated in Microsoft Power Point (2010). The finalized alignment and tree were deposited in TreeBASE, submission ID: 20319 (http://www.treebase.org/).

Table 2 GenBank and culture collection accession numbers of species included in the phylogenetic study. The newly generated sequence is shown in red bold. The ex-type strains are in black bold.

\begin{tabular}{|c|c|c|c|c|c|}
\hline \multirow[t]{2}{*}{ Species name } & \multirow[t]{2}{*}{ Strains no. } & \multicolumn{4}{|c|}{ GenBank accession number } \\
\hline & & LSU & SSU & ITS & tef1- $\alpha$ \\
\hline Alloconiothyrium aptrootii & CBS 980.95 & JX496234 & - & JX496121 & - \\
\hline Alloconiothyrium aptrootii & CBS 981.95 ${ }^{\mathrm{T}}$ & JX496235 & - & JX496122 & - \\
\hline Austropleospora archidendri & CBS $168.77^{T}$ & JX496162 & - & JX496049 & - \\
\hline Austropleospora osteospermi & LM 2009a $a^{T}$ & - & - & FJ481946 & - \\
\hline Bimuria novae-zelandiae & CBS 107.79 & AY016356 & AY016338 & - & - \\
\hline Deniquelata barringtoniae & MFLUCC 11-0422 ${ }^{\mathrm{T}}$ & JX254655 & JX254656 & JX254654 & - \\
\hline Deniquelata barringtoniae & MFLUCC 11-0257 ${ }^{\mathrm{T}}$ & KM213997 & KM214000 & KM214003 & - \\
\hline Didymocrea sadasivanii & CBS 438.65 & DQ384103 & DQ384066 & - & - \\
\hline Didymosphaeria rubi-ulmifolii & MFLUCC 14-0023 ${ }^{T}$ & KJ436586 & KJ436588 & KJ436586 & - \\
\hline Didymosphaeria rubi ulmifolii & CBS 100299 & JX496124 & AY642523 & JX496011 & - \\
\hline Didymosphaeria sp. & CBS 58784 & JX496212 & & JX496099 & - \\
\hline Kalmusia ebuli & CBS $1^{23120}{ }^{T}$ & JN644073 & JN851818 & - & - \\
\hline Kalmusia italica & MFLUCC 14-0560 ${ }^{T}$ & KP744487 & KP753953 & KP744441 & - \\
\hline Kalmusia spartii & MFLUCC 13-0352 ${ }^{T}$ & KM658315 & KM658316 & KM658314 & - \\
\hline Kalmusia variisporum & CBS $121517^{\mathrm{T}}$ & JX496143 & - & $\mathbf{J X 4 9 6 0 3 0}$ & - \\
\hline Karstenula rhodostoma & CBS 690.94 & GU301821 & GU296154 & - & - \\
\hline Karstenula rhodostoma & CBS 691.94 & AB807531 & AB797241 & - & AB808506 \\
\hline Laburnicola hawksworthii & MFLUCC 13-0602 ${ }^{\mathrm{T}}$ & KU743195 & KU743196 & KU743194 & - \\
\hline Laburnicola muriformis & MFLUCC 16-0290 & KU743198 & KU743199 & KU743197 & KU743213 \\
\hline Laburnicola muriformis & MFLUCC 14-0921 ${ }^{\mathrm{T}}$ & KU743201 & KU743202 & KU743200 & - \\
\hline Letendraea cordylinicola & MFLUCC 11-0148 & KM213995 & KM213998 & KM214001 & - \\
\hline Letendraea cordylinicola & MFLUCC 11-0150 ${ }^{T}$ & KM213996 & KM213999 & KM214002 & - \\
\hline Letendraea helminthicola & CBS 884.85 & AY016362 & AY016345 & - & - \\
\hline Letendraea padouk & CBS 485.70 & AY849951 & - & - & - \\
\hline Montagnula aloes & CPC $19671^{\mathrm{T}}$ & JX069847 & - & JX069863 & - \\
\hline Montagnula appendiculata & CBS $109027^{T}$ & AY772016 & - & DQ435529 & - \\
\hline Montagnula bellevaliae & MFLUCC 14-0924 ${ }^{\mathrm{T}}$ & KT443902 & KT443904 & KT443906 & - \\
\hline Montagnula cirsii & MFLUCC 13-0680 & KX274249 & KX274255 & KX274242 & KX284707 \\
\hline Montagnula donacina & HVVV01 & KJ628377 & KJ628376 & KJ628375 & - \\
\hline Montagnula graminicola & MFLUCC 13-0352 ${ }^{\mathrm{T}}$ & KM658315 & KM658316 & KM658314 & - \\
\hline Montagnula jonesii & MFLUCC 16-1448 & KY273276 & KY313618 & KY313619 & KY313620 \\
\hline Montagnula opulenta & CBS 168.34 & NG027581 & NG 013127 & AF383966 & - \\
\hline Montagnula saikhuensis & MFLUCC 16-0315 & KU743210 & KU743211 & KU743209 & - \\
\hline Montagnula scabiosae & MFLUCC 14-0954 ${ }^{T}$ & KT443903 & KT443905 & КT443907 & - \\
\hline Neokalmusia brevispora & KT $2313^{T}$ & AB524601 & AB524460 & - & AB539113 \\
\hline Neokalmusia didymospora & MFLUCC 11-0613 ${ }^{T}$ & КР091434 & KP091435 & KP091433 & - \\
\hline Neokalmusia scabrispora & KT 2202 & AB524594 & AB524453 & - & AB539107 \\
\hline Paracamarosporium fagi & CPC 24892 & KR611905 & - & KR611887 & - \\
\hline Paracamarosporium fagi & CPC 24890 & KR611904 & - & KR611886 & - \\
\hline Paracamarosporium hawaiiense & CBS $120025^{T}$ & JX496140 & EU295655 & JX496027 & - \\
\hline Paracamarosporium psoraleae & CPC $21632^{\mathrm{T}}$ & KF777199 & - & KF777143 & - \\
\hline Paraconiothyrium cyclothyrioides & CBS 972.95 ${ }^{\mathrm{T}}$ & JX496232 & AY642524 & JX496119 & - \\
\hline Paraconiothyrium estuarinum & CBS 109850 ${ }^{T}$ & JX496129 & AY642522 & JX496016 & - \\
\hline Paraconiothyrium fungicola & CBS $113269^{T}$ & JX496133 & AY 642527 & JX496020 & - \\
\hline Paramassariosphaeria clematidicola & MFLU 16-0172 ${ }^{\mathrm{T}}$ & KU743207 & KU743208 & KU743206 & - \\
\hline Paramassariosphaeria anthostomoides & CBS 615.86 & GU205223 & GU205246 & - & - \\
\hline Paraphaeosphaeria angularis & CBS $167.70^{\mathrm{T}}$ & JX496160 & - & JX496047 & - \\
\hline Paraphaeosphaeria michotii & MFLUCC 13-0349 & KJ939282 & KJ939285 & KJ939279 & - \\
\hline Paraphaeosphaeria minitans & CBS 111750 & JX496130 & - & JX496017 & - \\
\hline Paraphaeosphaeria minitans & CBS 859.71 & JX496229 & - & JX496116 & - \\
\hline
\end{tabular}




\begin{tabular}{llllll}
\hline Species name & Strains no. & \multicolumn{4}{c}{ GenBank accession number } \\
\cline { 3 - 6 } & & LSU & SSU & ITS & tef1- $\boldsymbol{\alpha}$ \\
\hline Phaeodothis winteri & AFTOL-ID 1590 & DQ678073 & DQ678021 & - & DQ677917 \\
Phaeodothis winteri & CBS 182.58 & GU301857 & GU296183 & - & - \\
Pleospora herbarum & CBS 191.86T & GU238160 & GU238232 & NR111243 & KC584731 \\
Pleospora herbarum & IT 956 & KP334709 & KP334729 & KP334719 & - \\
Pleospora trada & CBS 714.68T & KC584345 & KC584603 & KC584238 & KC584729 \\
Pseudocamarosporium corni & MFLUCC 13-0541 & KJ813279 & KJ819946 & KJ747048 & - \\
Pseudocamarosporium cotinae & MFLUCC 14-0624 & KP744505 & KP753964 & KP744460 & - \\
Pseudocamarosporium lonicerae & MFLUCC 13-0532 & KJ813278 & KJ819947 & KJ747047 & - \\
Pseudocamarosporium propinquum & MFLUCC 13-0544 & KJ813280 & KJ819949 & KJ747049 & - \\
Pseudopithomyces chartarum & UTHSC 04-678 & HG518065 & - & HG518060 & - \\
Pseudopithomyces chartarum & UTHSC 03-2472 & HG518064 & - & HG518059 & - \\
Pseudopithomyces sp. & MUCL 15905 & LK936383 & - & LK936375 & - \\
Pseudopithomyces sp. & MUCL 4329 & LK936382 & - & LK936374 & - \\
Spegazzinia deightonii & yone 212 & AB807582 & AB797292 & - & AB808558 \\
Spegazzinia sp. & yone 279 & AB807583 & AB797293 & - & AB808559 \\
Spegazzinia tessarthra & SH 287 & AB807584 & AB797294 & JQ673429 & AB808560 \\
Tremateia arundicola & MFLU 16-1275 & KX274248 & KX274254 & KX274241 & KX284706 \\
Tremateia guiyangensis & GZAAS01T & KX274247 & KX274253 & KX274240 & KX284705 \\
Tremateia halophila & JK 5517J & - & GU296201 & - & - \\
Verrucoconiothyrium nitidae & CBS 119209 & EU552112 & - & EU552112 & - \\
Xenocamarosporium acaciae & CPC 24755 & KR476759 & - & KR476724 & - \\
\hline
\end{tabular}

Abbreviations of culture collections: AFTOL-ID: Assembling the Fungal Tree of Life, CBS: Centraalbureau voor Schimmelcultures, Utrecht, The Netherlands, CPC: Working collection of Pedro Crous housed at CBS, GZAAS: Guizhou Academy of Agricultural Sciences herbarium, China, JK: J. Kohlmeyer, KT: K. Tanaka, LM: Secçáo de Botânica e Ecologia, Mozambique. MAPUTO, MFLU: Mae Fah Luang University, Chiang Rai, Thailand, MFLUCC: Mae Fah Luang University Culture Collection, Chiang Rai, Thailand, MUCL: Université Catholique de Louvain, Belgium, SH: Academia Sinica People's Republic of China. Shanghai, UTHSC: Fungus Testing Laboratory, University of Texas Health Science Center, San Antonio, Texas, USA, Yone: H. Yonezawa.

\section{Results}

Phylogenetic analysis

The combined LSU, SSU, ITS and tef1- $\alpha$ sequence data were analyzed with Pleospora herbarum (CBS 191.86, IT 956) and Pleospora tarda (CBS 714.68) as the outgroup taxa. The data set comprised 72 taxa including Montagnula jonesii. The maximum parsimony dataset comprised 3261 characters, including 2475 constant characters, 184 variable parsimony-uninformative characters and 602 parsimony-informative characters. The most parsimonious tree is shown where $\mathrm{TL}=2023, \mathrm{CI}=0.519, \mathrm{RI}=0.724, \mathrm{RC}=0.375, \mathrm{HI}=0.481$. Kishino-Hasegawa tests $(\mathrm{KHT})$ (Kishino \& Hasegawa 1989) were performed in order to determine whether trees were significantly different. Maximum likelihood (ML), maximum parsimony (MP) and Bayesian posterior probability analyses (PP) resulted in trees with similar topologies that did not differ significantly from one another (data not shown). The final RAxML tree is shown in Fig. 1, with the final ML optimization likelihood value of $-15278.83918(\mathrm{ln})$. The phylogeny showed that Montagnula jonesii grouped in Montagnula with strong support (99\% ML, 70\% MP and $0.99 \mathrm{PP})$, sister to M. saikhuensis (MFLUCC 16-0315), M. donacina (HVVV01) and M. graminicola (MFLUCC 13-0352). All analyses (ML, MP and PP) gave similar results of the generic placements in agreement with previous studies based on multi-gene analyses (Hyde et al. 2016, Li et al. 2016, Wanasinghe et al. 2016).

\section{Taxonomy}

Montagnula jonesii Tennakoon, Camporesi, Phookamsak \& K.D. Hyde, sp. nov. Index Fungorum number: IF552577; Facesoffungi number: FoF02719, Fig. 2 Holotype - MFLU 16-1363 


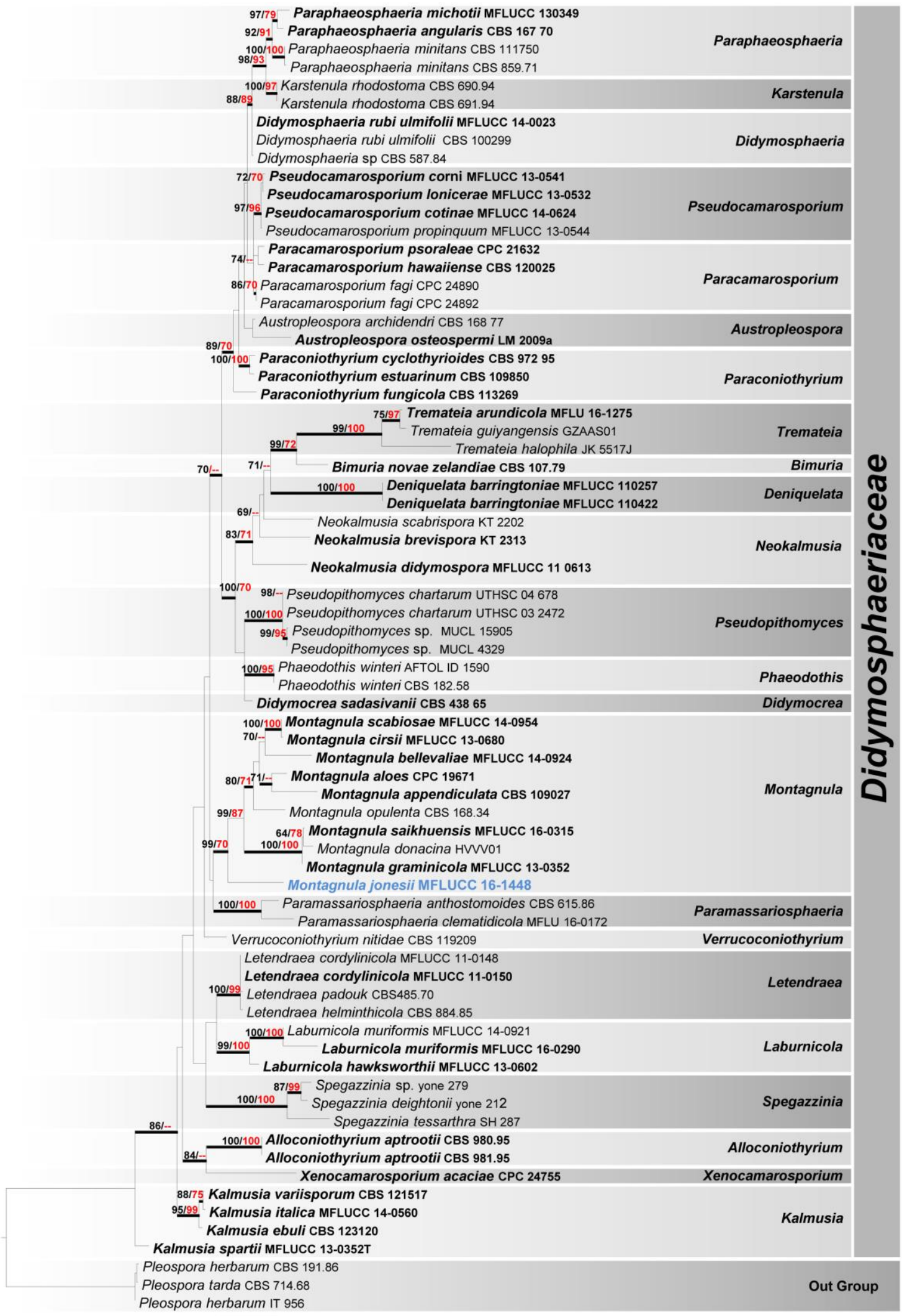

0.03

Fig. 1 - RAxML tree based on analysis of a combined LSU, SSU, ITS and tef1- $\alpha$ partial sequences. Bootstrap support values for maximum parsimony (MP, red) and maximum likelihood (ML, black) greater than $70 \%$ are defined above the nodes. Bayesian posterior probabilities (PP) greater than 0.90 are shown as bold branches. The tree is rooted to Pleospora herbarum (CBS 191.86, IT 956) and Pleospora tarda (CBS 714.68). The new strain is shown in blue. Ex-type strains are shown in bold. 
mycology

Etymology - In honour of Professor E.B. Gareth Jones for his immense contribution to

Saprobic on dead branches of Fagus sylvatica L. Sexual morph: Ascomata 140-210 $\mu \mathrm{m}$ diam., solitary, scattered to clustered, immersed or erumpent through host surface, visible as slightly raised, brown spots on host surface, globose to subglobose, glabrous, uniloculate, ostiole central with minute papilla. Peridium 10-25 $\mu \mathrm{m}$ wide, thin-walled with equal thickness, slightly thin at the base, composed of two layers of pseudoparenchymatous cells, inner layer comprising several cell layers of flattened, hyaline cells, arranged in a textura prismatica, outer layer comprising several layers of dark brown to black cells, arranged in a textura angularis. Hamathecium composed of 2-2.5 $\mu \mathrm{m}$ wide, dense, broad, filamentous pseudoparaphyses, distinctly septate, not constricted at the septum, anastomosing at the apex, embedded in a hyaline, gelatinous matrix. Asci (53-)60-70(-83) $\times(7.6-) 9-10(-11.5) \mu \mathrm{m}(\bar{x}=66.8 \times 9.5 \mu \mathrm{m}, \mathrm{n}=35)$, 8-spored, bitunicate, fissitunicate, clavate, long pedicellate, apically rounded, with well-developed ocular chamber. Ascospores $14-16(-17) \times 4-5.5 \mu \mathrm{m}(\bar{x}=15.5 \times 5 \mu \mathrm{m}, \mathrm{n}=35)$, overlapping 1-2-seriate, initially hyaline to pale brown, becoming brown to reddish-brown at maturity, ellipsoidal to fusiform with rounded ends, 1 -septate when young, becoming 3-septate when mature, constricted at the septa, straight to curved, enlarge at the second cell from apex, smooth-walled, with guttules. Asexual morph: Undetermined.

Culture characteristics - Colonies on PDA fast growing, reaching 7-8 cm diam. after two weeks at $20-25{ }^{\circ} \mathrm{C}$, colonies medium sparse, circular, flat, surface slightly rough with edge entire, margin well-defined, cottony to fairly fluffy with sparse aspects, colony from above, white to cream at the margin, light brown at the centre; from below, white brown to yellowish brown at the margin, mycelium green to grey with tufting; not producing pigmentation in PDA.

Material examined - ITALY, Arezzo Province (AR), near Croce di Pratomagno, on aerial and dead branches of Fagus sylvatica (Fagaceae), 21 June 2015, E. Camporesi, IT 2545 (MFLU 16-1363, holotype; HKAS93702, isotype), ex-type living cultures, MFLUCC 16-1448, KUMCC 15-0556.

Notes: Montagnula jonesii resembles to M. aloes Crous and M. scabiosae in having reddish-brown, 3-septate ascospores and immersed ostiolate ascomata. Montagnula jonesii has an unique character that can be used to distinguish it from $M$. aloes and $M$. scabiosae as ascospores have an enlarged second cell from the apex. Additionally, the size of asci and ascospores are different in each species (Table 3). Montagnula jonesii has ellipsoidal to fusiform ascospores, while they are ellipsoidal to ovoid in M. aloes. Montagnula jonesii is deeply constricted at septa, whereas M. scabiosae is slightly constricted (Table 3). Furthermore, each species is associated with a different host species (Table 3). A synopsis of the characters of species of Montagnula are provided in Table 3.

\section{Discussion}

Montagnula species play a vital role as saprobes growing on dead plants, especially dead wood and bark, sometimes on dead leaves (Ariyawansa et al. 2014b). Host-specificity of the taxa in this group have not yet been clarified according to they have been recorded from various plant families (i.e. Agavaceae, Arecaceae, Asparagaceae, Caprifoliaceae, Fagaceae, Poaceae, Xanthorrhoeaceae) (Table 3). Species of Montagnula seem to be cosmopolitan in distribution since they have been recorded from both temperate and tropical countries (i.e. Algeria, Australia, Italy, South Africa, Thailand) (Aptroot 1995, Wanasinghe et al. 2016). At the present, a well-resolved revision of the genus Montagnula is difficult since it lacks molecular data. From the 32 epithets present in Index Fungorum, there have been only 45 sequences from 12 species available in GenBank. The type species, $M$. infernalis (Niessl) Berl. does not have molecular data to verify its generic status and some sequences are not represented from the ex-type cultures, such as $M$. anthostomoides (Rehm) Leuchtm. (CBS 615.86), M. opulenta (De Not.) Aptroot (CBS 168.34). The connectively of sexual and as asexual morphs is not proven yet, as nobody has obtained any asexual morph for these new species from an ex-type culture which has molecular data. Also there 
is no molecular support to link possible asexual taxa. Therefore, representative species of these Montagnula species are essentially needed to be recollected and obtained molecular data for clarifying its phylogenetic affinity (especially from $M$. infernalis).

Table 3 Synopsis of recorded Montagnula species discussed in this study

\begin{tabular}{|c|c|c|c|c|c|c|}
\hline \multirow{2}{*}{$\begin{array}{l}\text { Montagnula } \\
\text { species }\end{array}$} & \multicolumn{3}{|c|}{ Size $(\mu \mathrm{m})$} & \multirow{2}{*}{$\begin{array}{l}\text { Septa in } \\
\text { ascospores }\end{array}$} & \multirow{2}{*}{ Host } & \multirow{2}{*}{ References } \\
\hline & Ascomata (diam.) & Asci & Ascospores & & & \\
\hline M. aloes & 450 & $110-250 \times 20-30$ & $33-36 \times 13-14$ & 3 & \multirow{3}{*}{$\begin{array}{l}\text { Aloe sp. } \\
\text { Zea mays } \\
\text { Bellevalia } \\
\text { romana }\end{array}$} & \multirow{3}{*}{$\begin{array}{l}\text { Crous et al. } 2012 \\
\text { Aptroot } 2004 \\
\text { Hongsanan et al. } \\
2015\end{array}$} \\
\hline M. appendiculata & $100-200$ & - & $12-15 \times 4-5$ & 1 & & \\
\hline M. bellevaliae & $100-120 \times 150-175$ & $70-100 \times 9-12$ & $15-18 \times 5-6$ & 2 & & \\
\hline M. cirsii & $385-415 \times 510-525$ & $\begin{array}{l}84.5-119.5 \times \\
10.5-13.5\end{array}$ & $\begin{array}{l}18-23.5 \times 6.5- \\
9.5\end{array}$ & 3 & Cirsium sp. & Hyde et al. 2016 \\
\hline M. donacina & - & - & $12-17 \times 4-6.5$ & 1 & Arundo donax & Aptroot 1995 \\
\hline M. graminicola & $37-117.22$ & $50-132 \times 8-13$ & $\begin{array}{l}9.8-13 \times 3.8- \\
5.5\end{array}$ & 1 & Grass & Liu et al. 2015 \\
\hline M. jonesii & $325-350 \times 300-325$ & $72-95 \times 9-13$ & $14-16 \times 5-6$ & 3 & $\begin{array}{l}\text { Fagus } \\
\text { sylvatica }\end{array}$ & This study \\
\hline M. opulenta & $400-1200$ & - & $19-25 \times 9-13$ & 1 & Opuntia sp. & Aptroot 1995 \\
\hline M. saikhuensis & $400-450 \times 400-500$ & $70-100 \times 10-12$ & $12-16 \times 4-6$ & 1 & Citrus sp. & $\begin{array}{l}\text { Wanasinghe et al. } \\
2016\end{array}$ \\
\hline M. scabiosae & $300-320 \times 300-360$ & $110-130 \times 14-20$ & $20-23 \times 7-9$ & 3 & Scabiosa sp. & $\begin{array}{l}\text { Hongsanan et al. } \\
2015\end{array}$ \\
\hline
\end{tabular}

\section{Acknowledgments}

The authors extend their sincere appreciations to the Deanship of Scientific Research at King Saud University for its funding this Prolific Research Group (PRG-1436-09). K.D Hyde thanks the Chinese Academy of Sciences, (project number 2013T2S003), for the award of visiting Professorship for Senior International Scientists at Kunming Institute of Botany. CGIAR Research Program 6: Forest, Trees and Agroforestry and Key Laboratory of Biodiversity and Biogeography, Kunming Institute of Botany, Chinese Academy of Sciences are thanked to the help of DNA molecular experiments. MFLU grant number 56101020032 is thanked for supporting studies on Dothideomycetes. R. Phookamsak thanks the Royal Golden Jubilee Ph.D Program (PHD/0090/2551) under the Thailand Research Fund for financial support. S. Khan thankful to National Natural Science Foundation China for the support of this work (Grant No. 31550110215). D. Tennakoon is grateful to K. Thambugala, QJ Shang, M. Samarakoon and N. de Silva for their valuable suggestions.

\section{References}

Aptroot A. 1995 - Redisposition of some species excluded from Didymosphaen'a (Ascomycotina). Nova Hedwigia 60, 325-379.

Aptroot A. 2004 - Two new ascomycetes with long gelatinous appendages collected from monocots in the tropics. Studies in Mycology 50, 307-312.

Ariyawansa HA, Camporesi E, Thambugala KM, Mapook A et al. 2014a - Confusion surrounding Didymosphaeria phylogenetic and morphological evidence suggest Didymosphaeriaceae is not a distinct family. Phytotaxa 176, 102-119.

Ariyawansa HA, Tanaka K, Thambugala KM, Phookamsak R et al. 2014b - A molecular phylogenetic reappraisal of the Didymosphaeriaceae (= Montagnulaceae). Fungal Diversity 68, 69-104.

Ariyawansa HA, Hyde KD, Jayasiri SC, Buyck B et al. 2015 - Fungal Diversity Notes 111-252taxonomic and phylogenetic contributions to fungal taxa. Fungal Diversity 75, 27-274.

Barr ME. 1990 - Some dictyosporous genera and species of Pleosporales in North America. Memoirs of the New York Botanical Garden 62, 1-92. 


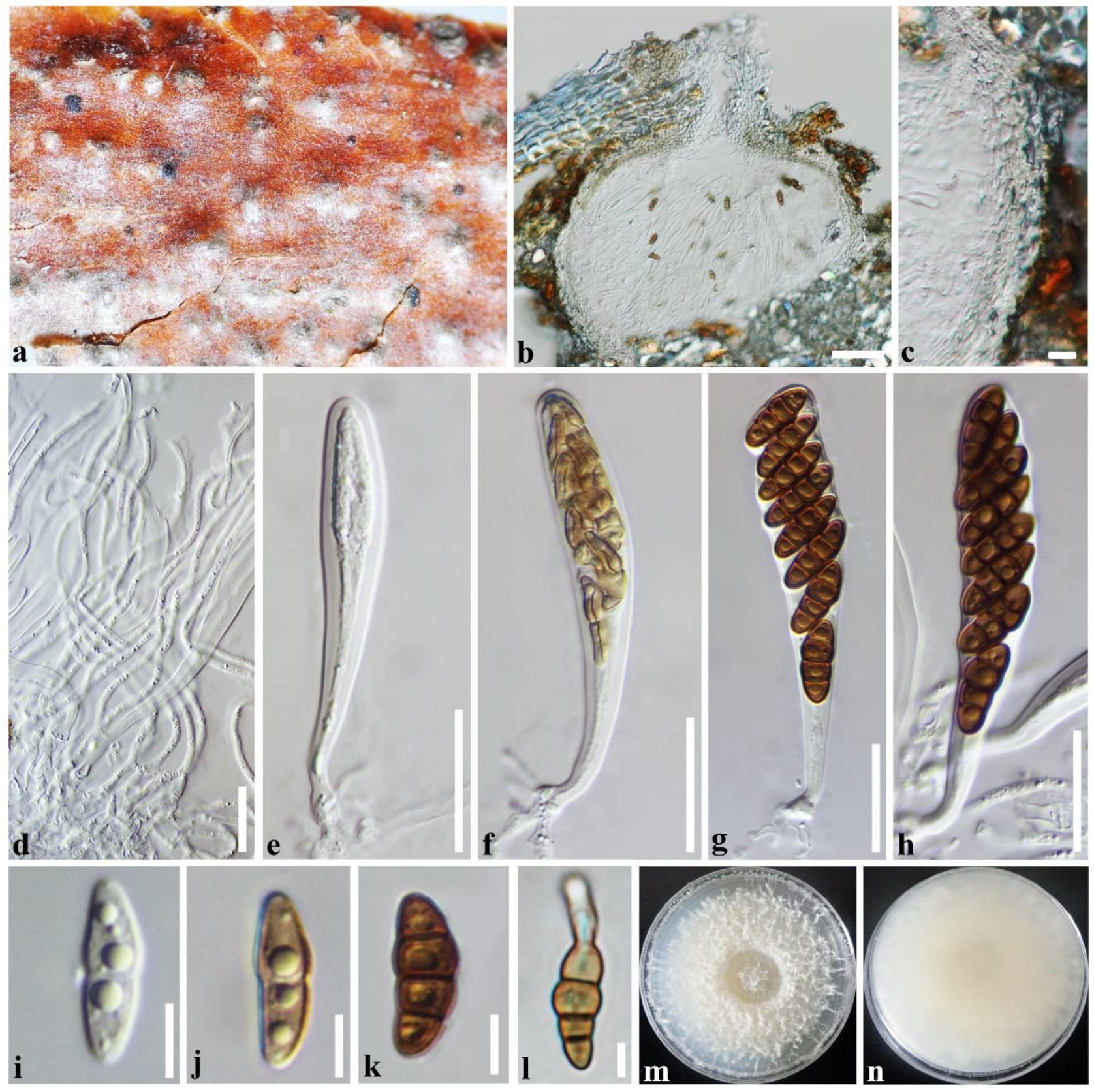

Fig. 2 - Montagnula jonesii (MFLU 16-1363, holotype). a Ascomata visible as black dots on the host surface. b Vertical section of ascoma. c Section through peridium. d Pseudoparaphyses. e-h Asci i-k Ascospores. 1 Germinated ascospore. $\mathrm{m}$ Colony from above. $\mathrm{n}$ Colony from below. Scale bars: $\mathrm{b}=50 \mu \mathrm{m}, \mathrm{c}=10 \mu \mathrm{m}, \mathrm{d}-\mathrm{l}=5 \mu \mathrm{m}$.

Berlese, A.N. 1896. Icones Fungorum. Pyrenomycetes. 2, 1-216.

Cai L, Jeewon R, Hyde KD. 2006 - Molecular systematics of Zopfiella and allied genera: evidence from multi-gene sequence analyses. Mycological Research 110, 359-368.

Crous PW, Schumacher RK, Wingfield MJ, Lombard L et al. 2015a - Fungal systematics and evolution: FUSE 1. Sydowia 67, 81-118.

Crous PW, Summerell BA, Shivas RG, Burgess TI et al. 2012 - Fungal Planet description sheets: 107-127. Persoonia 28, 138-182.

Crous PW, Wingfield MJ, Guarro J, Hernandez-Restrepo M et al. 2015b - Fungal Planet description sheets: 320-370. Persoonia 34, 167-266.

Hall TA. 1999 - BioEdit: a user- friendly biological sequence alignment editor and analysis program for Windows 95/98/NT. Nucleic Acids Symposium Series 41, 95-98. 
Hongsanan S, Hyde KD, Bahkali AH, Camporesi E et al. 2015 - Fungal Biodiversity Profiles 1120, Cryptogamie, Mycologie 36, 355-380.

Huelsenbeck JP, Ronquist F. 2001 - MRBAYES: Bayesian inference of phylogenetic trees. Bioinformatics 17, 754-755.

Hyde KD, Jones EBG, Liu JK, Ariyawansa H et al. 2013 - Families of Dothideomycetes. Fungal Diversity $63,1-313$.

Hyde KD, Jones EBG, Camporesi E, McKenzie EHC et al. 2016 - Fungal diversity notes 367-490: taxonomic and phylogenetic contributions to fungal taxa. Fungal Diversity 80, 1-270.

Index Fungorum 2016 - http://www.indexfungorum.org/Names/Names.asp.

Jayasiri CS, Hyde KD, Ariyawansa HA, Bhat DJ et al. 2015 - The Faces of Fungi database: fungal names linked with morphology, phylogeny and human impacts. Fungal Diversity 74, 3-18.

Kishino H, Hasegawa M. 1989 - Evaluation of the maximum likelihood estimate of the evolutionary tree topologies from DNA - sequence data, and the branching order in Hominoidea. Journal of Molecular Evolution 29, 170-179.

Li GJ, Hyde KD, Zhao RL, Hongsanan S et al. 2016 - Fungal diversity notes 253-366: taxonomic and phylogenetic contributions to fungal taxa. Fungal Diversity 78, 1-237.

Liu JK, Hyde KD, Jones EBG, Ariyawansa HA et al. 2015 - Fungal diversity notes 1-110: taxonomic and phylogenetic contributions to fungal species. Fungal Diversity 72, 1-197.

Liu JK, Phookamsak R, Doilom M, Wikee S et al. 2012 - Towards a natural classification of Botryosphaeriales. Fungal Diversity 57, 149-210.

Liu YJ, Whelen S, Hall BD. 1999 - Phylogenetic relationships among ascomycetes: evidence from an RNA polymerase II subunit. Molecular Biology and Evolution 16, 1799-1808.

Munk A. 1953 - The system of the pyrenomycetes. A contribution to a natural classification of the group Sphaeriales sensu Lindau. Dansk Botanisk Arkiv 15, 1-163.

Nylander JAA. 2004 - MrModeltest v2. Uppsala, Sweden: Evolutionary Biology Centre, Uppsala University.

Rambaut A. 2012 - FigTree version 1.4.0 Available at http://tree.bio.ed.ac.uk/software/figtree. Accessed November 2016.

Rannala B, Yang Z. 1996 - Probability distribution of molecular evolutionary trees: a new method of phylogenetic inference. Journal of Molecular Evolution 43, 304-311.

Rehner S. 2001 - Primers for elongation factor 1- $\alpha$ (EF1- $\alpha)$. http://www.aftol.org/pdfs/EF1primer.pdf

Silvestro D, Michalak I. 2012 - raxmlGUI: a graphical front-end for RAxML. Organisms Diversity and Evolution 12, 335-337.

Stamatakis A, Alachiotis N. 2010 - Time and memory efficient likelihood-based tree searches on phylogenomic alignments with missing data. Bioinformatics 26, 132-139.

Sung GH, Hywel-Jones NL, Sung JM, Luangsa-ard JJ et al. 2007 - Phylogenetic classification of Cordyceps and the clavicipitaceous fungi. Studies in Mycology 57, 5-59.

Swofford DL. 2002 - PAUP: Phylogenetic analysis using parsimony. Version 4.0 b10, Sinauer Associates, Sunderland.

Vilgalys R, Hester M. 1990 - Rapid genetic identification and mapping of enzymatically amplified ribosomal DNA from several Cryptococcus species. Journal of Bacteriology 172, 4238 4246.

Wanasinghe DN, Jones EBG, Camporesi E, Boonmee S et al. 2014 - An exciting novel member of Lentitheciaceae in Italy from Clematis vitalba. Cryptogamie Mycologie 35, 323-337.

Wanasinghe DN, Jones EBG, Camporesi E, Dissanayake AJ et al. 2016 - Taxonomy and phylogeny of Laburnicola gen. nov. and Paramassariosphaeria gen. nov. (Didymosphaeriaceae, Massarineae, Pleosporales). Fungal Biology 120, 1354-1373.

White T, Bruns T, Lee S, Taylor J. 1990 - Amplification and direct sequencing of fungal ribosomal RNA genes for phylogenetics. PCR Protocols: A Guide to Methods and Applications 18, $315-322$. 
Wijayawardene NN, Crous PW, Kirk PM, Hawksworth DL et al. 2014a - Naming and outline of Dothideomycetes - 2014 including proposals for the protection or suppression of generic names. Fungal Diversity 69, 1-55.

Wijayawardene NN, Hyde KD, Bhat DJ, Camporesi E et al. 2014b - Camarosporium-like species are polyphyletic in Pleosporales; introducing Paracamarosporium and Pseudocamarosporium gen. nov. in Montagnulaceae. Cryptogamie Mycologie 35, 177-198.

Zhaxybayeva O, Gogarten JP. 2002 - Bootstrap, Bayesian probability and maximum likelihood mapping: exploring new tools for comparative genome analyses. BMC genomics 3, 4. 